\title{
Using EELS to Determine He Pressure Inside Nanometer-Scale Bubbles
}

Joshua D. Sugar ${ }^{1}$, Ray D. Twesten ${ }^{2}$, Norman C. Bartelt ${ }^{1}$, Caitlin A. Taylor ${ }^{3}$, Noelle R. Catarineu ${ }^{1}$, and David B. Robinson ${ }^{1}$

1. Sandia National Laboratories, Livermore, CA, USA.

2. Gatan Inc., Pleasanton, CA, USA.

3. Sandia National Laboratories, Albuquerque, NM, USA.

He bubbles are a common form of radiation damage that degrade mechanical and electrical properties. They occur during ion implantation in $\mathrm{Si}$ [1,2], nuclear waste disposal [3], in nuclear reactors [4, 5], and in tritium storage materials [6]. Knowledge of the mechanisms of the initial bubble formation and their evolution is critical to predicting long-term performance of materials subjected to these harsh environments. The use of the TEM and EELS provides a unique way to characterize the spatial distribution and properties of these individual bubbles because the TEM's high-spatial resolution can reveal bubbles even at the nanoscale, and EELS' low-energy sensitivity enables He concentration measurements of the $21 \mathrm{eV} \mathrm{He} \mathrm{K}$ absorption line that can be directly converted to bubble pressure $[5,7]$. The ability to investigate correlations between bubble size and pressure is critical to testing theories of the mechanisms of the initial nucleation and eventual growth of these bubbles.

Here, we will investigate techniques for measuring the He concentration and pressure of bubbles in $\mathrm{Pd}$ alloys. Figure 1 demonstrates an example of He bubbles in $10 \mathrm{keV}$ ion-irradiated $\mathrm{Pd}\left(1 \times 10^{17}\right.$ ions $\left./ \mathrm{cm}^{2}\right)$ after being annealed in vacuum for $2 \mathrm{~h}$ at $600^{\circ} \mathrm{C}$. In this case, a simple windowed mapping can be used to identify the He bubbles, and the shift of the He peak to higher energies is due to the increased $\mathrm{He}$ pressure in these bubbles $(\sim 1.5 \mathrm{GPa})[5,7]$. Figure 2 shows another example in a Pd-5 at. \% Ni alloy that was tritiated and aged for 3.8 years. In this case, the bubbles are $\sim 5 \mathrm{~nm}$ in diameter, and no clear $\mathrm{He}$ peak can be delineated from the underlying plasmon peak. A simple least-squares fit with a blue-shifted He reference can identify only some of the bubbles. We will discuss more refined fitting routines we have developed to extract He concentration in these small-scale, high-pressure bubbles with the end goal of correlating pressure to bubble size. We will discuss our results in the context of models of He bubble formation and growth [8].

\section{References:}

[1] K. Alix, et al., Micron 77 (2015).

[2] R. Schierholz, et al., Nanotechnology 26 (2015).

[3] A.M. Seydoux-Guillaume, et al., Earth and Planetary Science Letters 448 (2016).

[4] M. Klimenkov, et al., Micron 46 (2013).

[5] D. Taverna, et al., Physical Review Letters 100 (2008).

[6] A. Fabre, et al., Journal of Nuclear Materials 342 (2005).

[7] S.E. Donnelly, Radiation Effects 90 (1985).

[8] Sandia National Laboratories is a multimission laboratory managed and operated by National Technology and Engineering Solutions of Sandia LLC, a wholly owned subsidiary of Honeywell International Inc., for the U.S. Department of Energy's National Nuclear Security Administration under contract DE-NA0003525. 


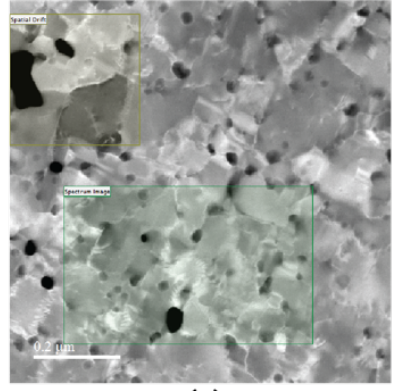

(a)

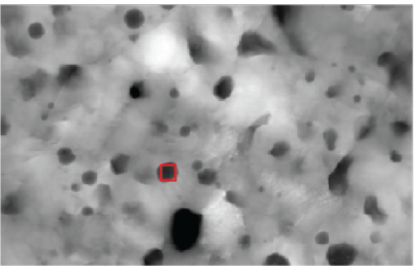

(b)

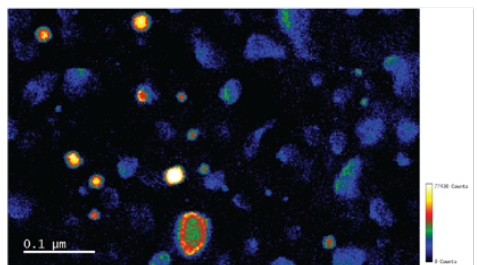

(c)

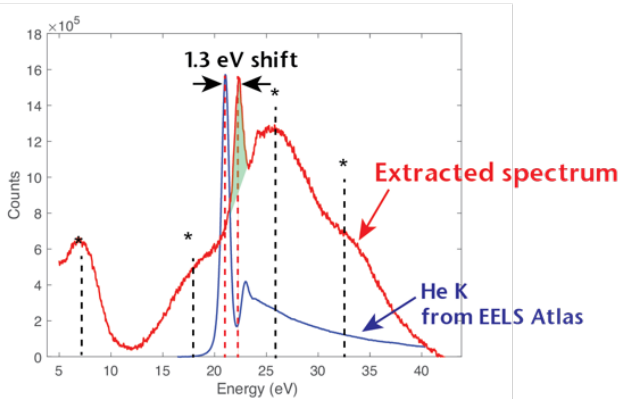

(d)

Figure 1. (a) ADF STEM image of $10 \mathrm{keV}$ He-ion implanted $\mathrm{Pd}\left(1 \times 10^{17}\right.$ ions $\left./ \mathrm{cm}^{2}\right)$ after annealing $2 \mathrm{~h}$ at $600^{\circ} \mathrm{C}$, (b) HAADF signal collected during spectrum image of green boxed region in (a), (c) conventional "window" signal map of green energy region in (d). In (d), the He K reference from the EELS Atlas and the sum spectrum from the red boxed region in (b) are compared. The peaks with asterisks correspond to the plasmon peaks for $\mathrm{Pd}$ at 7, 17, 26, and $33 \mathrm{eV}$.

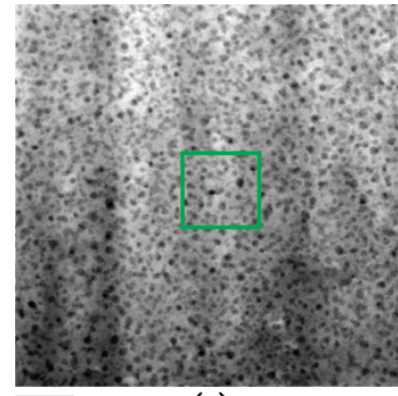

$\overline{20 \mathrm{~nm}}$

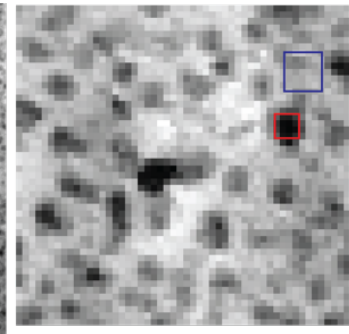

(b)
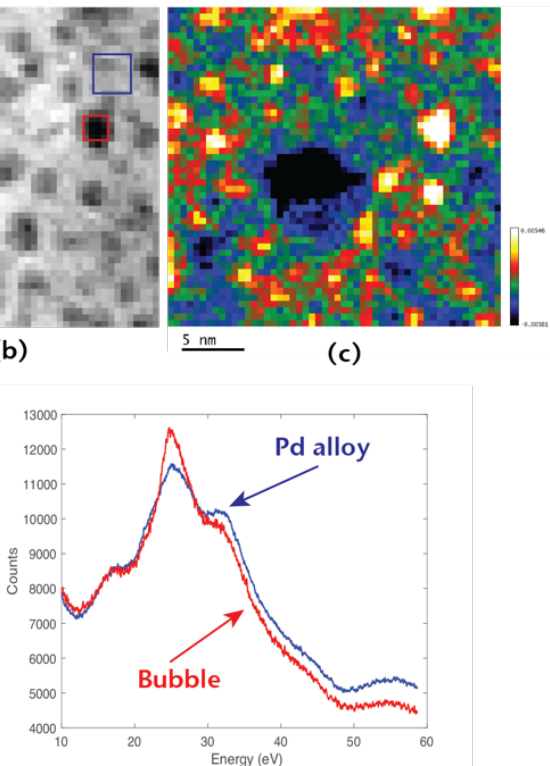

(d)

Figure 2. (a) ADF STEM image of He bubbles in tritiated Pd-Ni alloy aged for 3.8 years at room temperature, (b) ADF signal collected during spectrum image of green boxed region in (a), (c) MLLS fit to blue-shifted He K reference signal from the EELS Atlas and blue boxed region in (b) showing identification of some of the bubbles. In (d), sum spectra from the blue boxed and red boxed regions in (b) are compared to show the difference between bubble spectra and Pd-alloy spectra. 